\title{
Three-week combination chemotherapy with S-1 and cisplatin as first-line treatment in patients with advanced gastric cancer: a retrospective study with 159 patients
}

\author{
Dong Hoe Koo $\cdot$ Min-Hee Ryu $\cdot$ Baek-Yeol Ryoo $\cdot$ Sung-Sook Lee $\cdot$ Jung-Hwa Moon $\cdot$ \\ Heung-Moon Chang • Jae-Lyun Lee · Tae Won Kim • Yoon-Koo Kang
}

Received: 28 June 2011/Accepted: 31 October 2011/Published online: 10 December 2011

(C) The International Gastric Cancer Association and The Japanese Gastric Cancer Association 2011

\begin{abstract}
Background Doses and schedules of the combination of S-1 and cisplatin for the treatment of advanced gastric cancer (AGC) have not been standardized. We therefore evaluated the efficacy and feasibility of a 3-week schedule of S-1 and cisplatin in patients with AGC, as well as assessing factors prognostic of patient outcomes.

Methods A total of 159 patients with AGC were treated with S-1 (40 mg/ $\mathrm{m}^{2}$ bid on days $\left.1-14\right)$ and cisplatin $\left(60 \mathrm{mg} / \mathrm{m}^{2} \mathrm{IV}\right.$ on day 1) between January 2004 and December 2008.

Results Median follow-up duration was 20.0 months (range, 11.4-48.5 months), during which time 129 patients $(81.1 \%)$ died. Patients received a median 6 cycles of chemotherapy (range, 1-19 cycles). Among the 59 patients with measurable disease, 1 achieved a complete response $(1.7 \%)$ and $24(40.7 \%)$ had partial responses, giving an overall response rate of $42.4 \%$ (95\% CI, 23.0-61.8\%). The median progression-free survival (PFS) was 5.8 months (95\% CI, 4.8-6.9 months), and the median overall survival (OS) was 11.3 months (95\% CI, 9.6-13.0 months). Multivariate analysis showed that initial metastasis, bone metastasis, and liver metastasis were independent prognostic factors for reduced PFS, whereas poor performance status, initial metastasis, and bone metastasis were prognostic for reduced OS. Application of a previous prognostic model showed that observed PFS and OS survival curves
\end{abstract}

D.H. Koo and M.-H. Ryu contributed equally to the work as first authors.

D. H. Koo · M.-H. Ryu B.-Y. Ryoo - S.-S. Lee · J.-H. Moon · H.-M. Chang · J.-L. Lee · T. W. Kim · Y.-K. Kang ( $\bowtie)$ Department of Oncology, Asan Medical Center, University of Ulsan College of Medicine, 86 Asanbyeongwon-gil, Songpa-gu, Seoul 138-736, Korea e-mail: ykkang@amc.seoul.kr for patients in various risk groups differed significantly $(P<0.001$ each $)$.

Conclusions A 3-week regimen of S-1 plus cisplatin was active and well tolerated as first-line treatment in patients with AGC. Disease status and bone metastasis were the most important prognostic factors.

Keywords Stomach neoplasms $\cdot$ S-1 $\cdot$ Cisplatin . Chemotherapy · Prognosis

\section{Introduction}

Gastric cancer is the second leading cause of cancer death worldwide, with patients who are diagnosed at an advanced stage having a median survival time of less than 1 year [1]. Several randomized clinical trials showed that, compared with best supportive care alone, chemotherapy plus best supportive care improved median patient survival, from 4-6 months to 9-11 months, and the quality of life in patients with advanced gastric cancer (AGC) [2, 3]. Moreover, a large meta-analysis showed that first-line treatment with combination chemotherapy resulted in a survival benefit compared with monotherapy [hazard ratio (HR), 0.82; 95\% confidence interval (CI), 0.74-0.90] [4]. Most chemotherapy regimens for AGC are based on a fluoropyrimidine-plus-platinum backbone, with or without an anthracycline [5].

$\mathrm{S}-1$ is a compound drug consisting of the oral fluoropyrimidine prodrug, tegafur; the dihydropyrimidine dehydrogenase (DPD) inhibitor, 5-chloro-2,4-dihydroxypyridine (CDHP, gimeracil); and the orotate phosphoribosyl transferase (OPRT) inhibitor, potassium oxonate (OXO, oteracil) [6]. Several studies in Japan showed that S-1 monotherapy or S-1 plus cisplatin had antitumor activity against AGC [7, 8]. 
Two recent phase III trials in Japan showed that S-1 monotherapy was not inferior to the continuous infusion of 5-fluorouracil (5-FU) and that $\mathrm{S}-1$ plus cisplatin was superior to S-1 monotherapy $[9,10]$. Based on these results, the combination of $\mathrm{S}-1$ with cisplatin became the new standard treatment for AGC in Japan.

Because a standardized schedule of S-1 and cisplatin in combination has not been established, several doses and schedules were evaluated $[8,11,12]$. Although a 5-week schedule ( 3 weeks on and 2 weeks off) has been used primarily in Japan [10], the dose of cisplatin was relatively lower in this schedule than in other phase III studies [13, 14]. A phase I/II study of S-1, consisting of 2 weeks of treatment followed by 1 week of rest with a fixed cisplatin dosage, as first-line chemotherapy for AGC showed encouraging results [15]. The recommended doses of S-1 and cisplatin were determined as $80 \mathrm{mg} / \mathrm{m}^{2} /$ day and $60 \mathrm{mg} / \mathrm{m}^{2}$, respectively, and this 3-week schedule has been utilized in clinical practice in Korea. We have therefore evaluated the efficacy and safety of this 3-week schedule of S-1 plus cisplatin in patients with AGC. In addition, we analyzed factors prognostic of patient outcome and evaluated whether our previously reported prognostic model was applicable to these patients [16].

\section{Materials and methods}

\section{Patients}

We retrospectively reviewed the Asan Medical Center Stomach Cancer Registry to identify all patients who had been treated for AGC at the Asan Medical Center (Seoul, Korea) between January 2004 and December 2008. We included patients 18 years of age and older with histologically confirmed recurrent or metastatic adenocarcinoma of the stomach; with adequate bone marrow, renal, and hepatic function; who received at least one cycle of chemotherapy; and who had no history of other malignancies. A total of 1,925 patients with recurrent or metastatic gastric cancer received first-line chemotherapy, and 159 patients (8.3\%) were identified to receive this 3 -week schedule of S-1 with cisplatin chemotherapy as first-line treatment of AGC. The study protocol was approved by the Institutional Review Board of the Asan Medical Center.

Patients were grouped into three categories according to disease status at the time of chemotherapy initiation: those with initially metastatic disease (i.e., presenting with metastatic disease), those with recurrent disease (i.e., presenting with tumor recurrence after curative gastrectomy), and those with resected metastatic disease (i.e., presenting with residual disease after gastrectomy or with distant metastases who had gastrectomies) [17].
In addition, patients were grouped by our prognostic model for recurrent or metastatic AGC [16]. This model used a scoring system consisting of eight prognostic factors [Eastern Cooperative Oncology Group (ECOG) performance score ( $\mathrm{PS}) \geq 2$ ( 2 points); no gastrectomy, peritoneal metastasis, or bone metastasis ( 2 points); and lung metastasis, alkaline phosphatase $>120 \mathrm{IU} / \mathrm{l}$, albumin $<3.3 \mathrm{~g} / \mathrm{dl}$, or total bilirubin $>1.2 \mathrm{mg} / \mathrm{dl}$ ], with patients divided into good ( $0-1$ points), moderate ( $2-3$ points), and poor $(\geq 4$ points) risk groups.

\section{Treatment schedule}

Before chemotherapy, all patients were evaluated with a complete history and physical examination, a complete blood count, serum chemistry evaluations (liver and renal function tests and electrolytes), ECG, chest radiograph, computed tomography (CT) scanning of the abdomen and pelvis, and, if indicated, CT scans of the chest and a bone scan. All patients were treated with $40 \mathrm{mg} / \mathrm{m}^{2} \mathrm{~S}-1$, administered orally twice daily on days $1-14$; and $60 \mathrm{mg} / \mathrm{m}^{2}$ cisplatin, administered intravenously over $60 \mathrm{~min}$ on day 1 . Treatment courses were repeated every 3 weeks. Treatment was continued until disease progression or unacceptable toxicity occurred, or if the patient chose to discontinue treatment.

\section{Dose modification for adverse events}

S-1 and/or cisplatin doses were modified for hematological, gastrointestinal, or neurological toxicities, based on the most severe grade of toxicity occurring during the previous cycle. Patients were assessed before the beginning of each cycle, in accordance with the National Cancer InstituteCommon Toxicity Criteria (NCI-CTC) version 3.0 (http://www.cancer.gov). When a grade 3 or 4 hematological toxicity occurred, except for anemia, or a grade 2 or 3 nonhematological toxicity occurred during a 2-week period of S-1 administration, S-1 was interrupted until the hematological toxicity subsided to grade 2 or the nonhematological toxicity subsided to grade 1 , with S-1 subsequently resumed at the same dose or reduced by $25 \%$, respectively. If there was a second occurrence of grade 4 hematological or grade 3 nonhematological toxicity after dose reduction, S-1 treatment was interrupted temporarily, and was resumed at $50 \%$ of the original dose. The subsequent chemotherapy cycle was started when absolute neutrophil count (ANC), platelet count, and nonhematological toxicities recovered. Cisplatin dose was modified according to renal toxicity and peripheral neuropathy. If serum creatinine before each cycle was $<1.5 \mathrm{mg} / \mathrm{dl}$, fulldose cisplatin was given; if serum creatinine was $1.5-2.5 \mathrm{mg} / \mathrm{dl}, 50 \%$ cisplatin was administered; if serum 
creatinine was $>2.5 \mathrm{mg} / \mathrm{dl}$, chemotherapy was discontinued. If a grade 2 neurotoxicity occurred, cisplatin treatment was delayed up to 3 weeks until neuropathy recovered to grade 1 or better. If neuropathy persisted for more than 3 weeks or a grade 3 or higher neuropathy occurred, chemotherapy was discontinued.

\section{Response evaluation}

Physical examination, chest X-rays, complete blood counts, and biochemical tests were performed before each chemotherapy cycle. CT scans were performed every two or three cycles until the tumor progressed. Tumor response was classified according to the response evaluation criteria in solid tumors (RECIST) 1.0 guidelines. Confirmation of the response was not required for this study.

\section{Statistical analysis}

Overall survival (OS) was measured from the starting date of chemotherapy until death from any cause. Progressionfree survival (PFS) was measured from the starting date of chemotherapy until tumor progression or death from any cause. Survival rates were estimated using the KaplanMeier method and compared using the log-rank test. Prognostic factors were analyzed by searching all clinical variables in univariate analysis, with all variables with a $P$ value $<0.10$ in the univariate analysis entered into multivariate analysis using stepwise Cox proportional hazard regression models. A two-sided $P$ value $<0.05$ was considered significant, and 95\% confidential intervals (CIs) were calculated. All statistical analyses were performed using the SPSS software package (SPSS, Chicago, IL, USA).

\section{Results}

Patient characteristics

Median age was 54 years (range, 29-84 years); of these, $98(61.6 \%)$ were men and $138(86.8 \%)$ had ECOG PS of 0 to 1 . Patients were followed up for a median 20.0 months (range, 11.4-48.5 months). Overall, 129 patients $(81.1 \%)$ died, with a median survival time of 11.3 months (95\% CI, 9.6-13.0 months). Patient characteristics are summarized in Table 1. Before the start of S-1 plus cisplatin chemotherapy, 24 patients (15.1\%) underwent palliative gastrectomy (resected metastatic group), $40(25.2 \%)$ had recurrent disease after curative gastrectomy (recurrent group), and 95 (59.7\%) had distant metastases without gastrectomy (initially metastatic group).
Table 1 Patient characteristics

\begin{tabular}{|c|c|}
\hline Characteristic & $n=159(100 \%)$ \\
\hline \multicolumn{2}{|l|}{ Age (years) } \\
\hline Median (range) & $54.0(29-84)$ \\
\hline \multicolumn{2}{|l|}{ Gender } \\
\hline Male & $98(61.6)$ \\
\hline Female & $61(38.4)$ \\
\hline \multicolumn{2}{|l|}{ ECOG PS } \\
\hline $0 / 1$ & $138(86.8)$ \\
\hline $2 / 3$ & $21(13.2)$ \\
\hline \multicolumn{2}{|l|}{ Histology } \\
\hline WD/MD & $30(18.9)$ \\
\hline $\mathrm{PD} / \mathrm{UD}$ & $106(66.7)$ \\
\hline NA & $23(14.5)$ \\
\hline \multicolumn{2}{|l|}{ Disease status } \\
\hline Resected metastatic & $24(15.1)$ \\
\hline Recurrent & $40(25.2)$ \\
\hline Metastatic & $95(59.7)$ \\
\hline \multicolumn{2}{|l|}{ Tumor location } \\
\hline Upper $1 / 3$ & $6(3.8)$ \\
\hline Middle $1 / 3$ & $52(32.7)$ \\
\hline Lower $1 / 3$ & $32(20.1)$ \\
\hline Multiple & $19(11.9)$ \\
\hline NA & $50(31.4)$ \\
\hline Measurable lesion (presence) & $59(37.1)$ \\
\hline \multicolumn{2}{|l|}{ Metastasis } \\
\hline Peritoneum & $93(58.5)$ \\
\hline Liver & $30(18.9)$ \\
\hline Abdominal lymph nodes & $48(30.2)$ \\
\hline Lung & $5(3.1)$ \\
\hline Bone & $9(5.7)$ \\
\hline \multicolumn{2}{|l|}{ Number of metastases } \\
\hline 1 & $115(72.3)$ \\
\hline$\geq 2$ & $44(27.7)$ \\
\hline Second-line chemotherapy (received) & $85(53.5)$ \\
\hline Albumin $(<3.3 \mathrm{~g} / \mathrm{dl})$ & $52(32.7)$ \\
\hline Alkaline phosphatase (>120 IU/l) & $26(16.4)$ \\
\hline Total bilirubin $(>1.2 \mathrm{mg} / \mathrm{dl})$ & $11(6.9)$ \\
\hline
\end{tabular}

ECOG PS Eastern Cooperative Oncology Group Performance Status, $W D$ well differentiated, $M D$ moderately differentiated, $P D$ poorly differentiated, $U D$ undifferentiated, $N A$ not available

Tumor response

Of the 59 patients with measurable disease, 5 were not evaluable for tumor response because they were lost to follow-up after the first treatment cycle (Table 2). Twentyfour patients $(40.7 \%)$ achieved a partial response (PR), with 1 achieving a complete response (CR, 1.7\%), making the overall response rate (ORR) $42.4 \% \quad(95 \%$ CI, 
Table 2 Response rate

\begin{tabular}{llll}
\hline Response & $\begin{array}{l}\text { Resected metastatic } \\
\text { or recurrent }(n=21)\end{array}$ & $\begin{array}{l}\text { Initially } \\
\text { metastatic } \\
(n=38)\end{array}$ & $\begin{array}{l}\text { Total (\%) } \\
(n=59)\end{array}$ \\
\hline Complete response & 0 & 1 & $1(1.7)$ \\
Partial response & 9 & 15 & $24(40.7)$ \\
Stable disease & 6 & 11 & $17(28.8)$ \\
Disease progression & 2 & 10 & $12(20.3)$ \\
Not evaluable & 4 & 1 & $5(8.5)$ \\
Response rate $(\%)$ & 42.9 & 42.1 & 42.4 \\
\hline
\end{tabular}

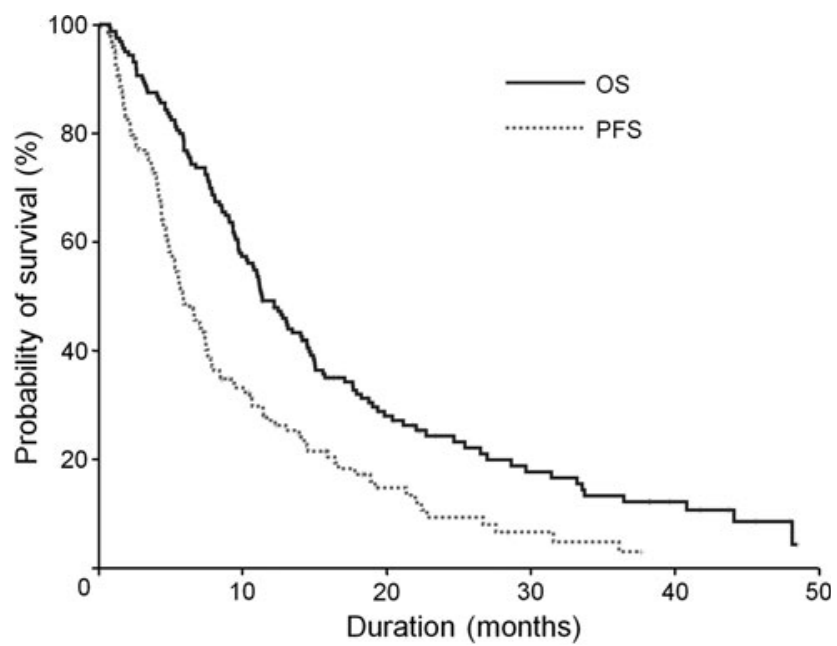

Fig. 1 Kaplan-Meier survival curves of progression-free survival $(P F S)$ and overall survival $(O S)$

23.0-61.8\%). The median duration of response in these 25 patients was 5.6 months (range, 3.4-7.9 months). There was no difference in response rate among the resected metastatic, recurrent, and initially metastatic groups $(\mathrm{RR}=$ $42.1,42.1$, and 50.0\%, respectively; $P=0.269$ ).

Survival outcome

The median PFS was 5.8 months (95\% CI, 4.8-6.9 months) and the median OS was 11.3 months (95\% CI, 9.6-13.0 months; Fig. 1). Both PFS and OS differed significantly among the three groups. The resected metastatic, recurrent, and initially metastatic groups had median PFS values of 14.7, 7.4, and 4.5 months, respectively $(P<0.001)$, and median OS values of 25.4, 15.0, and 9.3 months, respectively $(P<0.001$; Fig. 2$)$.

Adverse events

Patients received a median of 6 cycles of chemotherapy (range, 1-19 cycles). The most common treatment-related hematological adverse events were anemia (95.0\%) and
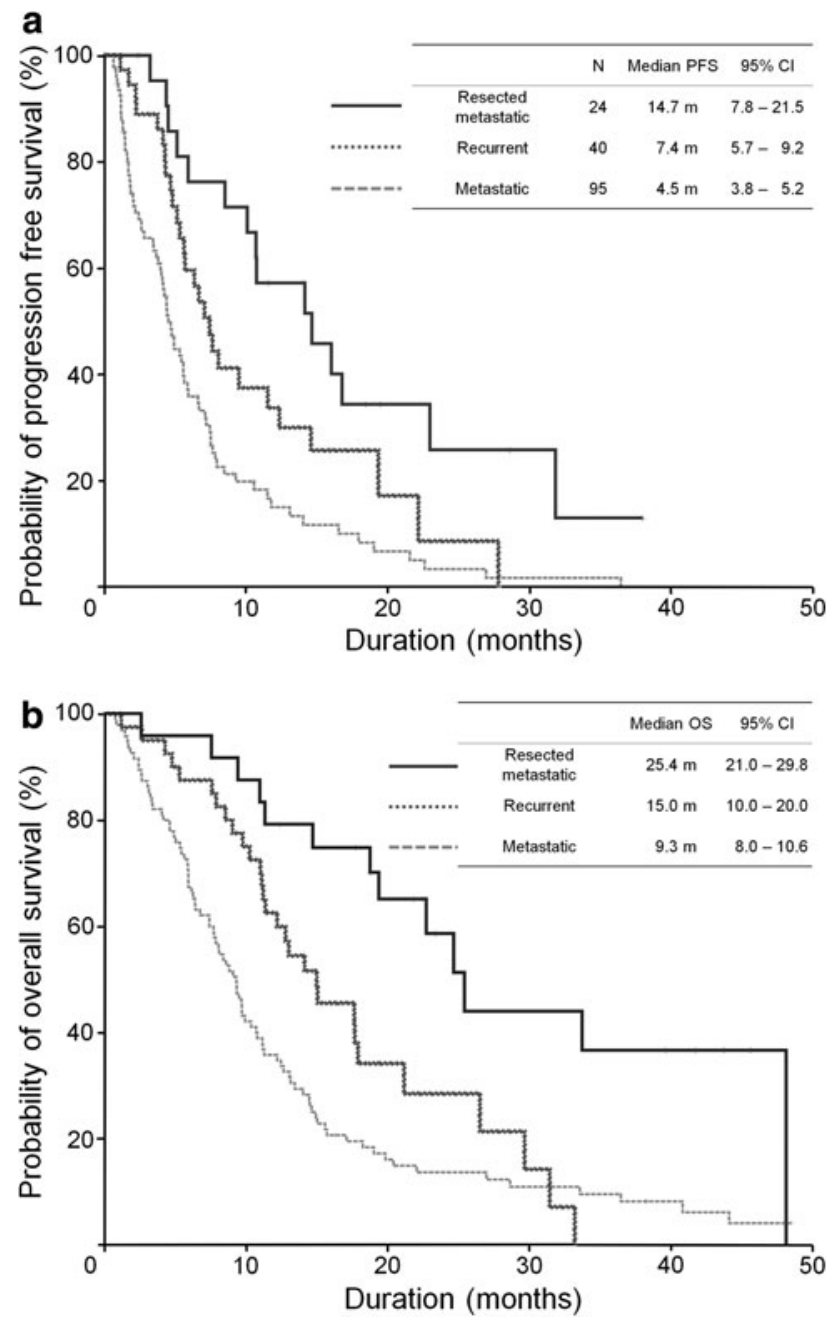

Fig. 2 Kaplan-Meier survival curves relative to disease status of progression-free survival (PFS) (a) and overall survival (OS) (b). $C I$, confidence interval

neutropenia (78.0\%), with grades 3 and 4 anemia occurring in $18.9 \%$ and $1.3 \%$ of these patients, respectively, and grades 3 and 4 neutropenia occurring in 31.4 and $10.1 \%$, respectively. Grade 3 thrombocytopenia was observed in 13 patients (8.2\%), but none had grade 4 thrombocytopenia. Nausea and vomiting were relatively common (16.3\%), but only 1 patient had grade 3 nausea and vomiting. Grade 3 diarrhea, stomatitis, anorexia, and fatigue were observed in 1.9, 1.3, 1.9, and $0.6 \%$ of patients, respectively. Drug dose was reduced in 49 patients (30.8\%): in 41 (83.7\%) for hematological toxicities, in 4 each $(8.2 \%)$ for anorexia and fatigue, and in 1 (2.0\%) for diarrhea. Treatment was delayed in 68 patients $(42.8 \%)$ : in $56(82.1 \%)$ for hematological toxicities, in $2(3.0 \%)$ for nausea or vomiting, and in $1(1.5 \%)$ for infection. During the first to third cycle of chemotherapy, 19 patients $(11.9 \%)$ required dose reductions and 35 (22.0\%) required treatment delays. In addition, median relative dose intensity was $87.9 \%$ for scheduled cycles during the first 6 cycles. 
Prognostic factors and prognostic model

Table 3 shows the results of univariate analysis of factors prognostic of patient survival. Performance status, disease status, number of metastases, peritoneal metastases, liver metastases, and bone metastases were significant or borderline prognostic factors for PFS or OS. Multivariate analysis showed that the initial metastatic group (vs. recurrent and resected metastatic group), bone metastasis, and liver metastasis were independent prognostic factors for reduced PFS (Table 4), and that poor PS, initial metastatic group, and bone metastasis were independent prognostic factors for reduced OS. After applying our prognostic model [16], we found that the survival curves of patients in various risk groups showed highly significant differences in both PFS and OS $(P<0.001$ each; Fig. 3). The good, moderate, and poor risk groups had a median PFS of 7.6, 5.3, and 2.6 months, respectively, and a median OS of 19.8, 10.8, and 6.7 months, respectively.

\section{Discussion}

We evaluated the efficacy and safety of a 3-week combination of S-1 and cisplatin in AGC patients in the clinical practice setting. We also identified factors prognostic of patient survival, and we applied our prognostic model to categorize these patients into three risk groups.
Table 3 Univariate analysis of factors predictive of prognosis
PFS progression-free survival, $O S$ overall survival, ECOG PS Eastern Cooperative Oncology Group Performance Status, WD well differentiated, $M D$ moderately differentiated, $P D$ poorly differentiated, $U D$ undifferentiated, $L N$ lymph node

\begin{tabular}{|c|c|c|c|c|c|c|c|}
\hline & $n$ & $\begin{array}{l}\text { Median } \\
\text { PFS (months) }\end{array}$ & $95 \% \mathrm{CI}$ & $P$ value & $\begin{array}{l}\text { Median } \\
\text { OS (months) }\end{array}$ & $95 \% \mathrm{CI}$ & $P$ value \\
\hline \multicolumn{8}{|l|}{ Age (years) } \\
\hline$\leq 54$ & 81 & 5.1 & $4.0-6.3$ & \multirow[t]{2}{*}{0.609} & 11.4 & $9.1-13.6$ & \multirow[t]{2}{*}{0.813} \\
\hline$>54$ & 78 & 7.2 & $5.1-9.2$ & & 11.2 & $7.9-14.6$ & \\
\hline \multicolumn{8}{|l|}{ Gender } \\
\hline Male & 98 & 6.7 & $4.8-8.6$ & \multirow[t]{2}{*}{0.707} & 11.3 & $9.3-13.4$ & \multirow[t]{2}{*}{0.558} \\
\hline Female & 61 & 5.6 & $4.1-7.2$ & & 11.3 & $7.4-15.2$ & \\
\hline \multicolumn{8}{|l|}{ ECOG PS } \\
\hline $0 / 1$ & 138 & 6.6 & $5.3-7.9$ & \multirow[t]{2}{*}{0.012} & 12.6 & $10.3-15.0$ & \multirow[t]{2}{*}{0.001} \\
\hline $2 / 3$ & 21 & 3.9 & $1.6-6.3$ & & 6.7 & $4.9-8.6$ & \\
\hline \multicolumn{8}{|l|}{ Histology } \\
\hline WD/MD & 30 & 6.7 & $3.0-10.3$ & \multirow[t]{2}{*}{0.798} & 13.1 & $7.6-18.7$ & \multirow[t]{2}{*}{0.111} \\
\hline $\mathrm{PD} / \mathrm{UD}$ & 106 & 5.7 & $4.6-6.8$ & & 10.7 & $8.5-12.9$ & \\
\hline \multicolumn{8}{|l|}{ Disease status } \\
\hline Resected meta & 24 & 14.7 & $7.8-21.5$ & \multirow[t]{3}{*}{$<0.001$} & 25.4 & $21.0-29.8$ & \multirow[t]{3}{*}{$<0.001$} \\
\hline Recurrent & 40 & 7.4 & $5.7-9.2$ & & 15.0 & $10.0-20.0$ & \\
\hline Metastatic & 95 & 4.5 & $3.8-5.2$ & & 9.3 & $8.0-10.6$ & \\
\hline \multicolumn{8}{|l|}{ No. of metastases } \\
\hline 1 & 115 & 7.1 & $5.4-8.8$ & \multirow[t]{2}{*}{0.028} & 13.4 & $10.5-16.4$ & \multirow[t]{2}{*}{0.002} \\
\hline$\geq 2$ & 44 & 4.9 & $3.7-6.1$ & & 9.3 & $8.3-10.4$ & \\
\hline \multicolumn{8}{|c|}{ Peritoneum metastasis } \\
\hline Yes & 93 & 5.4 & $3.9-7.0$ & \multirow[t]{2}{*}{0.893} & 11.2 & $8.7-13.6$ & \multirow[t]{2}{*}{0.058} \\
\hline No & 66 & 6.7 & $5.2-8.2$ & & 14.1 & $10.0-18.3$ & \\
\hline \multicolumn{8}{|l|}{ Liver metastasis } \\
\hline Yes & 30 & 3.9 & $2.9-5.0$ & \multirow[t]{2}{*}{$<0.001$} & 10.3 & $7.7-13.0$ & \multirow[t]{2}{*}{0.316} \\
\hline No & 129 & 6.6 & $5.1-8.1$ & & 12.2 & $10.2-14.1$ & \\
\hline \multicolumn{8}{|l|}{ Lung metastasis } \\
\hline Yes & 5 & 7.6 & $0.0-16.0$ & \multirow[t]{2}{*}{0.905} & 17.6 & $7.3-28.0$ & \multirow[t]{2}{*}{0.203} \\
\hline No & 154 & 5.8 & $4.8-6.9$ & & 11.3 & $9.3-13.3$ & \\
\hline \multicolumn{8}{|c|}{ Abdominal LN metastasis } \\
\hline Yes & 48 & 5.6 & $4.9-6.2$ & 0.096 & 9.8 & 7.9-11.6 & 0.225 \\
\hline No & 111 & 6.6 & $4.9-8.3$ & & 12.6 & $10.8-14.5$ & \\
\hline Bone metastasis & & & & & & & \\
\hline Yes & 9 & 2.0 & $0.0-5.7$ & 0.004 & 9.7 & $9.1-10.3$ & 0.013 \\
\hline No & 150 & 5.9 & $4.5-7.3$ & & 12.2 & $10.0-14.4$ & \\
\hline
\end{tabular}


Table 4 Multivariate analysis of factors predictive of poor prognosis

\begin{tabular}{llllllrr}
\hline & & HR for PFS & $95 \%$ CI & $P$ value & HR for OS & $95 \%$ CI & $P$ value \\
\hline ECOG PS & $2 / 3$ & 1.76 & $0.98-3.14$ & 0.057 & 1.78 & $1.05-3.02$ & 0.034 \\
Initial status & Metastatic & 2.05 & $1.39-3.02$ & $<0.001$ & 2.19 & $1.51-3.18$ & $<0.001$ \\
Peritoneum metastasis & Yes & - & - & - & 1.36 & $0.95-1.96$ & 0.098 \\
Bone metastasis & Yes & 2.73 & $1.27-5.88$ & 0.010 & 2.09 & $1.01-4.32$ & 0.048 \\
Liver metastasis & Yes & 2.10 & $1.33-3.30$ & 0.001 & - & - \\
\hline
\end{tabular}

$H R$ hazard ratio, PFS progression-free survival, OS overall survival, ECOG PS Eastern Cooperative Oncology Group Performance Status
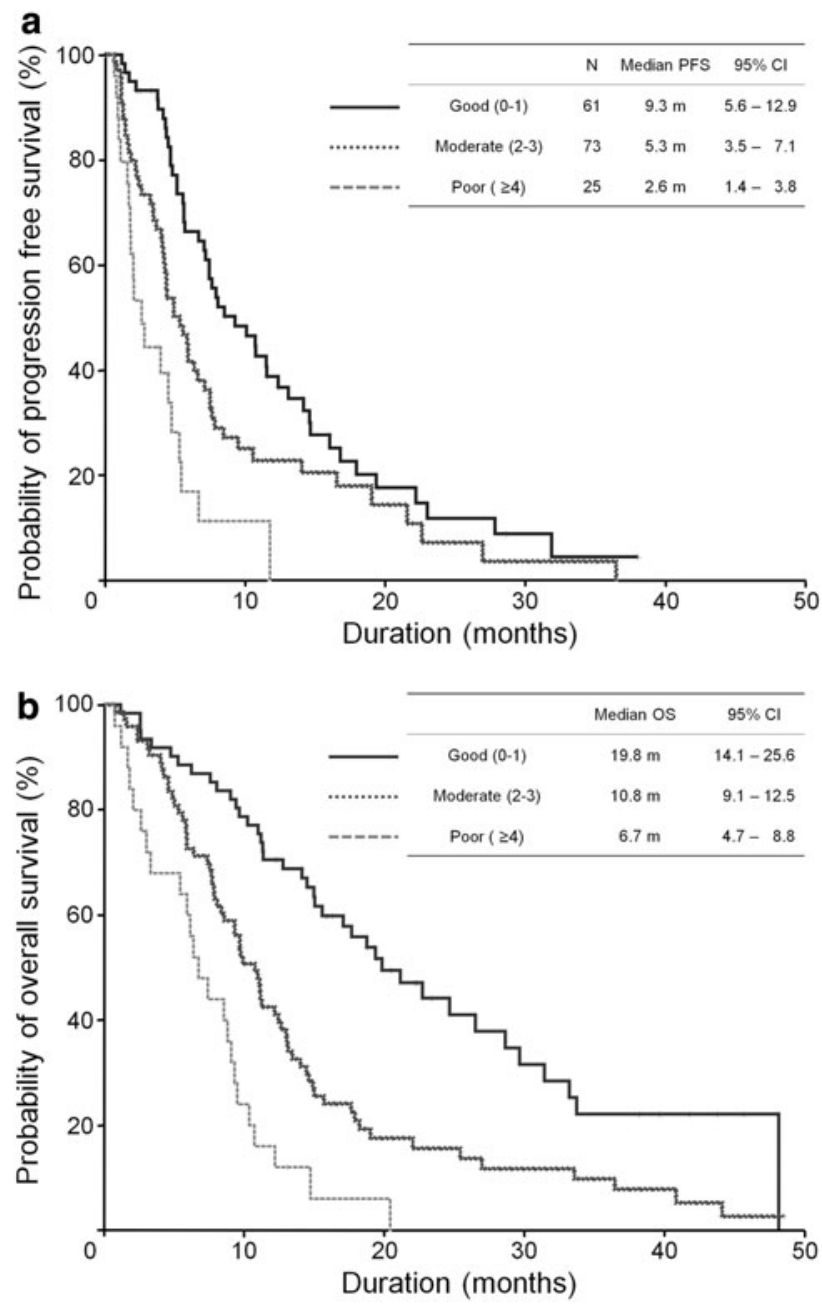

Fig. 3 Kaplan-Meier survival curves relative to our prognostic model of progression-free survival $(P F S)$ (a) and overall survival $(O S)(\mathbf{b})$

We observed an ORR of $42.4 \%$, a median PFS of 5.6 months, and a median OS of 11.4 months, similar to the results of our phase II study (confirmed RR, 47.6\%; median PFS, 5.3 months; median OS, 10.0 months) [15] and within the range of those observed in recent phase 3 trials of fluoropyrimidine and platinum-based agents as first-line chemotherapy in AGC patients (RR, 25-45\%; median PFS, 4-6 months; median OS, 8-11 months) [1821]. Our median survival, however, was shorter than that observed with a 5-week schedule in the Japanese SPIRITS trial. It is difficult to compare these trials directly, although a higher percentage of our patients had poorer PS (ECOG PS $2 / 3 ; 13$ vs. $3 \%$ ) and fewer had received second-line chemotherapy (54 vs. $74 \%$ ). In addition, the SPIRITS trial results require further confirmation. Our regimen resulted in generally acceptable safety and toxicity. Grade 1/2 hematological toxicities and nausea/vomiting were relatively common, but severe toxicities were rare, similar to results in our previous phase II study and SPIRITS trial $[10,15]$. Thus, our findings indicate that the 3-week schedule of combination S-1 with cisplatin is effective and safe in the treatment of AGC, not only in a clinical trial setting but also in clinical practice.

We identified four factors independently prognostic of poor PFS and OS, all of which had previously been associated with poor prognosis in patients with AGC: poor PS [17], initial metastatic (no prior gastrectomy) [17], liver metastasis [22], and bone metastasis [23]. Because disease status, particularly initial metastatic (vs. recurrent or resected metastatic), and bone metastasis were independently significant factors for both PFS and OS, these two factors seemed to be most representative of tumor burden in this patient population. When our prognostic model was applied [16], we found that the three risk groups had highly significant differences in PFS and OS $(P<0.001$ each), indicating the applicability of our model in this patient population.

To date, there have been many modified schedules for S-1 and cisplatin combination chemotherapy. Initially, S-1 was developed in Japan and usually administered in doses of 40-60 mg bid, depending on each patient's body surface area, in a 5-week cycle ( 3 weeks on and 2 weeks off), in combination with $60 \mathrm{mg} / \mathrm{m}^{2}$ cisplatin on day $8[8,10]$. However, a subsequent Japanese post-marketing survey of S-1 showed that this schedule resulted in median times to worst toxic events of 22 days for hematological toxicities and 15 days for diarrhea and stomatitis, with median recovery times of about 2 weeks from these toxicities [24]. These findings suggested that a 3-week cycle may have 
some advantages. In Western studies, S-1 was given for 3 weeks followed by a 1 -week rest; the maximal tolerated dose (MTD) of S-1 was $50 \mathrm{mg} / \mathrm{m}^{2} /$ day when given with cisplatin $75 \mathrm{mg} / \mathrm{m}^{2}$ on day 1 of each 4-week cycle [19, 25, 26]. The development of late-onset diarrhea was a major concern in these studies. Because most toxicities occurred during the third week of administration in both Japanese and Western studies, a drug-free interval in the third week of each cycle may reduce the incidence of adverse reactions and improve efficacy. Indeed, S-1 showed better toxicity profiles and similar efficacy when given in 3-week rather than 6-week cycles to patients with head and neck cancer [27].

The Japanese 5-week schedule resulted in a lower dose of cisplatin $\left(12 \mathrm{mg} / \mathrm{m}^{2} /\right.$ week $)$ [10] than with the reference dose $\left(20-25 \mathrm{mg} / \mathrm{m}^{2} /\right.$ week) for AGC in several phase III studies $[13,14]$. The S-1 dose was much lower in Western $\left(50 \mathrm{mg} / \mathrm{m}^{2} /\right.$ day) than in Asian $\left(70-80 \mathrm{mg} / \mathrm{m}^{2} /\right.$ day $)$ studies, which may be the result of differences in cytochrome P450 metabolism related to genetic polymorphisms in CYP2A6 [28]. Based on these findings, we conducted a phase I/II study of a 3 -week schedule of S-1 $\left(80 \mathrm{mg} / \mathrm{m}^{2} /\right.$ day $)$ plus cisplatin $\left(60 \mathrm{mg} / \mathrm{m}^{2}\right)$ [15]. These different schedules and dosages of S-1 plus cisplatin make it difficult to compare the results of these clinical trials, to choose the optimal schedule in clinical practice, and to design global trials using S-1 plus cisplatin with or without targeted agents. A prospective phase III trial of a 3-week versus a 5-week schedule of S-1 with cisplatin (SOS study) is currently underway in Korea and Japan.

In conclusion, the 3 -week combination of S-1 plus cisplatin was safe and effective as first-line treatment for patients with AGC in a clinical practice setting. Disease status and bone metastasis were the most important prognostic factors in these patients, and our prognostic model was able to classify patients into three groups with significantly different survival outcomes following treatment with S-1 plus cisplatin.

Conflict of interest None.

\section{References}

1. Hohenberger P, Gretschel S. Gastric cancer. Lancet. 2003;362: 305-15.

2. Pyrhonen S, Kuitunen T, Nyandoto P, Kouri M. Randomised comparison of fluorouracil, epidoxorubicin and methotrexate (FEMTX) plus supportive care with supportive care alone in patients with non-resectable gastric cancer. Br J Cancer. 1995;71:587-91.

3. Glimelius B, Ekström K, Hoffman K, et al. Randomized comparison between chemotherapy plus best supportive care with best supportive care in advanced gastric cancer. Ann Oncol. 1997;8:163-8.

4. Wagner AD, Unverzagt S, Grothe W, et al. Chemotherapy for advanced gastric cancer. Cochrane Database Syst Rev. 2010; CD004064.
5. Kang YK, Yoon DH, Ryoo BY, Ryu MH. Recent advances in chemotherapy for advanced gastric cancer. Asia Pac J Oncol Hematol 2010;2.

6. Shirasaka T, Shimamato Y, Ohshimo H, et al. Development of a novel form of an oral 5-fluorouracil derivative (S-1) directed to the potentiation of the tumor selective cytotoxicity of 5-fluorouracil by two biochemical modulators. Anti Cancer Drugs. 1996;7:548-57.

7. Sakata Y, Ohtsu A, Horikoshi N, Sugimachi K, Mitachi Y, Taguchi T. Late phase II study of novel oral fluoropyrimidine anticancer drug S-1 (1 M tegafur- $0.4 \mathrm{M}$ gimestat-1 M otastat potassium) in advanced gastric cancer patients. Eur $\mathrm{J}$ Cancer. 1998;34:1715-20.

8. Koizumi W, Tanabe S, Saigenji K, et al. Phase I//II study of S-1 combined with cisplatin in patients with advanced gastric cancer. Br J Cancer. 2003;89:2207-12.

9. Boku N, Yamamoto S, Fukuda H, et al. Fluorouracil versus combination of irinotecan plus cisplatin versus S-1 in metastatic gastric cancer: a randomised phase 3 study. Lancet Oncol. 2009;10:1063-9.

10. Koizumi W, Narahara H, Hara T, et al. S-1 plus cisplatin versus $\mathrm{S}-1$ alone for first-line treatment of advanced gastric cancer (SPIRITS trial): a phase III trial. Lancet Oncol. 2008;9:215-21.

11. Sato $\mathrm{Y}$, Kondo H, Honda $\mathrm{K}$, et al. A phase I/II study of S-1 plus cisplatin in patients with advanced gastric cancer: 2-week S-1 administration regimen. Int J Clin Oncol. 2005;10:40-4.

12. Kimura $\mathrm{Y}$, Kikkawa $\mathrm{N}$, Iijima $\mathrm{S}$, et al. A new regimen for $\mathrm{S}-1$ therapy aiming at adverse reaction mitigation and prolonged medication by introducing a 1-week drug-free interval after each 2-week dosing session: efficacy and feasibility in clinical practice. Gastric Cancer. 2003;6:34-9.

13. Vanhoefer U, Rougier P, Wilke H, et al. Final results of a randomized phase III trial of sequential high-dose methotrexate, fluorouracil, and doxorubicin versus etoposide, leucovorin, and fluorouracil versus infusional fluorouracil and cisplatin in advanced gastric cancer: a trial of the European Organization for Research and Treatment of Cancer Gastrointestinal Tract Cancer Cooperative Group. J Clin Oncol. 2000;18:2648-57.

14. Van Cutsem E, Moiseyenko VM, Tjulandin S, et al. Phase III study of docetaxel and cisplatin plus fluorouracil compared with cisplatin and fluorouracil as first-line therapy for advanced gastric cancer: a report of the V325 Study Group. J Clin Oncol. 2006;24:4991-7.

15. Lee J-L, Kang H, Kang Y-K, et al. Phase I/II study of 3-week combination of S-1 and cisplatin chemotherapy for metastatic or recurrent gastric cancer. Cancer Chemother Pharm. 2008;61: $837-45$.

16. Koo DH, Ryoo B-Y, Kim HJ, et al. A prognostic model in patients who receive chemotherapy for metastatic or recurrent gastric cancer: validation and comparison with previous models. Cancer Chemother Pharm 2011;1(1):87-95

17. Lee SS, Lee J-L, Ryu M-H, et al. Combination chemotherapy with capecitabine $(\mathrm{X})$ and cisplatin $(\mathrm{P})$ as first line treatment in advanced gastric cancer: experience of 223 patients with prognostic factor analysis. Jpn J Clin Oncol. 2007;37:30-7.

18. Kang Y-K, Kang W-K, Shin D-B, et al. Capecitabine/cisplatin versus 5-fluorouracil/cisplatin as first-line therapy in patients with advanced gastric cancer: a randomised phase III noninferiority trial. Ann Oncol. 2009;20:666-73.

19. Ajani JA, Rodriguez W, Bodoky G, et al. Multicenter phase III comparison of cisplatin/S-1 with cisplatin/infusional fluorouracil in advanced gastric or gastroesophageal adenocarcinoma study: the FLAGS trial. J Clin Oncol. 2010;28:1547-53.

20. Al-Batran S-E, Hartmann JT, Probst S, et al. Phase III trial in metastatic gastroesophageal adenocarcinoma with fluorouracil, leucovorin plus either oxaliplatin or cisplatin: a study of the 
Arbeitsgemeinschaft Internistische Onkologie. J Clin Oncol. 2008;26:1435-42.

21. Dank M, Zaluski J, Barone C, et al. Randomized phase III study comparing irinotecan combined with 5-fluorouracil and folinic acid to cisplatin combined with 5-fluorouracil in chemotherapy naive patients with advanced adenocarcinoma of the stomach or esophagogastric junction. Ann Oncol. 2008;19:1450-7.

22. Chau I, Norman AR, Cunningham D, Waters JS, Oates J, Ross PJ. Multivariate prognostic factor analysis in locally advanced and metastatic esophago-gastric cancer-pooled analysis from three multicenter, randomized, controlled trials using individual patient data. J Clin Oncol. 2004;22:2395-403.

23. Kim JG, Ryoo BY, Park YH, et al. Prognostic factors for survival of patients with advanced gastric cancer treated with cisplatinbased chemotherapy. Cancer Chemother Pharm. 2008;61:301-7.

24. Nagashima F, Ohtsu A, Yoshida S, Ito K. Japanese nationwide post-marketing survey of S-1 in patients with advanced gastric cancer. Gastric Cancer. 2005;8:6-11.
25. Ajani JA, Faust J, Ikeda K, et al. Phase I pharmacokinetic study of S-1 plus cisplatin in patients with advanced gastric carcinoma. J Clin Oncol. 2005;23:6957-65.

26. Ajani JA, Lee F-C, Singh DA, et al. Multicenter phase II trial of S-1 plus cisplatin in patients with untreated advanced gastric or gastroesophageal junction adenocarcinoma. J Clin Oncol. 2006; 24:663-7.

27. Tsukuda M, Kida A, Fujii M, et al. Randomized scheduling feasibility study of S-1 for adjuvant chemotherapy in advanced head and neck cancer. Br J Cancer. 2005;93:884-9.

28. Zhu A, Clark J, Ryan D, et al. Phase I and pharmacokinetic study of S-1 administered for 14 days in a 21-day cycle in patients with advanced upper gastrointestinal cancer. Cancer Chemother Pharm. 2007;59:285-93. 